Design of Flexure-based Precision Transmission

Mechanisms using Screw Theory

J. B. Hopkins, R. M. Panas

February 9, 2011

11th International Conference of the European Society for Precision Engineering \& Nanotechnology Como, Italy May 23, 2011 through May 27, 2011 
This document was prepared as an account of work sponsored by an agency of the United States government. Neither the United States government nor Lawrence Livermore National Security, LLC, nor any of their employees makes any warranty, expressed or implied, or assumes any legal liability or responsibility for the accuracy, completeness, or usefulness of any information, apparatus, product, or process disclosed, or represents that its use would not infringe privately owned rights. Reference herein to any specific commercial product, process, or service by trade name, trademark, manufacturer, or otherwise does not necessarily constitute or imply its endorsement, recommendation, or favoring by the United States government or Lawrence Livermore National Security, LLC. The views and opinions of authors expressed herein do not necessarily state or reflect those of the United States government or Lawrence Livermore National Security, LLC, and shall not be used for advertising or product endorsement purposes. 


\title{
Design of Flexure-based Precision Transmission Mechanisms using Screw Theory
}

J.B. Hopkins ${ }^{1}$, R.M. Panas ${ }^{2}$

${ }^{1}$ Lawrence Livermore National Laboratories USA

${ }^{2}$ Massachusetts Institute of Technology USA

\section{hopkins30@llnl.gov,rpanas@mit.edu}

\begin{abstract}
This paper enables the synthesis of flexure-based transmission mechanisms that possess multiple decoupled inputs and outputs of any type (e.g. rotations, translations, and/or screw motions), which are linked by designer-specified transmission ratios. A comprehensive library of geometric shapes is utilized from which every feasible concept that possesses the desired transmission characteristics may be rapidly conceptualized and compared before an optimal concept is selected. These geometric shapes represent the rigorous mathematics of screw theory and uniquely link a body's desired motions to the flexible constraints that enable those motions. This paper's impact is most significant to the design of nano-positioners, microscopy stages, optical mounts, and sensors. A flexure-based microscopy stage was designed, fabricated, and tested to demonstrate the utility of the theory.
\end{abstract}

\section{$1 \quad$ Introduction}

In this paper we introduce the theory that may be used to optimally synthesize complex flexure-based transmission mechanisms. These mechanisms are important to precision engineering because of their ability to amplify or attenuate an input displacement or load on a structure in a repeatable way. As such, they may be used as low-cost solutions for (i) increasing the resolution of a system's actuators, (ii) improving the sensitivity of a systems sensors, and (iii) transforming the nature of a system's inputs (e.g. from rotations to translations). Two sets of geometric shapes have been derived [1] that enable the synthesis of such mechanisms. One set represents the degrees of freedom (DOFs) a system possesses and the other set represents the constraints that enable those DOFs. Consider, for instance, the flexure shown Fig. 1A. This system possesses a single screw DOF shown as a line along 
which the stage may translate as it rotates according to the screw's pitch, $p$, shown in Fig. 1B. The screw line is uniquely linked to another shape of circular hyperboloids shown in Fig. 1C from which constraints may be selected that enable the screw motion. The axes of the constraints that lie on the surfaces of these hyperboloids all satisfy the screw line according to

$$
p=d \cdot \tan \varphi,
$$

where $d$ and $\varphi$ are defined in Fig. 1D. Note from Fig. 1E and Fig. 1F that the constraints of the flexure system shown in Fig. 1A all belong to the shape of Fig. 1C.

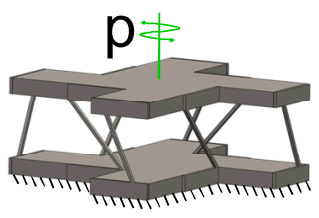

(A)

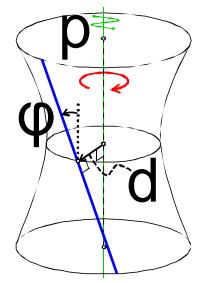

(D)

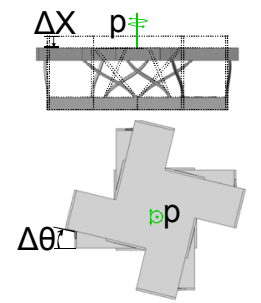

(B)

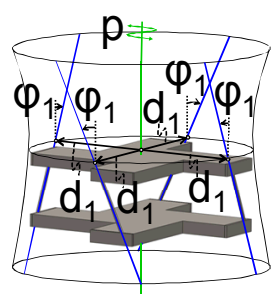

(E)

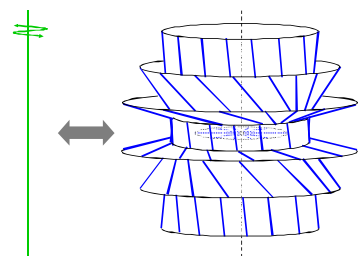

(C)

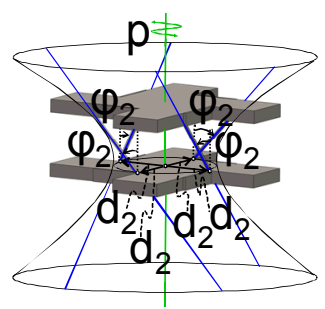

(F)

Figure 1: Using geometric shapes to synthesize flexure systems

\section{Principles of Flexure-based Transmission Synthesis}

Other flexure systems that are designed using geometric shapes may be attached to the systems that possess screw DOFs to transform their screw motions into desired transmissions. Suppose, for instance, a system that possessed a single translational DOF were attached to the top plate of the system from Fig. 1A, and a system that possessed a single rotational DOF were attached to the bottom plate as shown in Fig. 2A. If the constraints were ideal (i.e., infinitely stiff along their axes compared to other directions), the output stage would translate as the input stage rotated as shown 
in Fig. 2B with a transmission ratio equal to the pitch of the screw from Fig. 1A. The actual transmission ratio is always smaller than this pitch and may be found using

$$
\mathbf{T}_{\text {Output }}=\left[K_{T W}\right]_{1}^{-1}\left(\left[K_{T W}\right]_{1}^{-1}+\left[K_{T W}\right]_{2}^{-1}\right)^{-1} \mathbf{T}_{\text {Input }},
$$

where $\left[K_{T W}\right]_{1},\left[K_{T W}\right]_{2}$, and $\left[K_{T W}\right]_{3}$ are the $6 x 6$ twist-wrench stiffness matrices of each flexure module in the system and $\mathbf{T}_{\text {Output }}$ and $\mathbf{T}_{\text {Input }}$ are the $6 x 1$ twist vectors that describe the input and output displacements as shown in Fig. 2C. See Hopkins [2] to learn how to construct these matrices and vectors for any flexure and displacement. Note from Eq. (2) and from the analogue system shown in Fig. 2D that the twistwrench stiffness matrices obey the same rules for calculating transmission ratios as linear springs with scalar stiffness values. Note also that the system's transmission ratio will be different depending on which stage is actuated as the input.

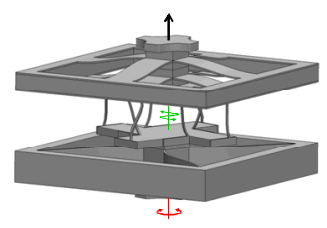

(A)

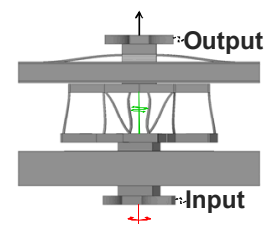

(B)

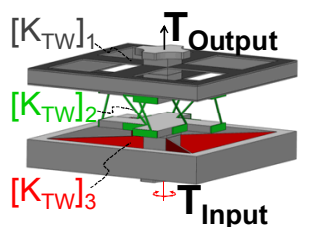

(C)

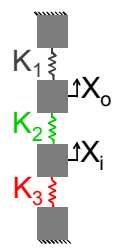

(D)

Figure 2: Synthesis of a flexure-based transmission mechanism using screw theory

\section{Synthesizing Multiple Decoupled Input Transmission Mechanisms}

The principles of this paper may be applied to the synthesis of transmission mechanisms with multiple decoupled inputs. Consider the flexure system from Fig. 3A that possesses two intersecting screw DOFs represented by a disk of screws. These screws share a common pitch, $p$, that may be found using Eq. (1) for the parameters labeled in Fig. 3A. The system's constraints lie on the surfaces of nested circular hyperboloids shown in Fig. 3B. A transmission ratio less than the pitch of these screws could be achieved by attaching flexure systems that would constrain the top plate of the system to rotate about the axes of the screw lines and the bottom plate to translate in the direction of the screw lines as shown in Fig. 3C. Examples of such flexure attachments are shown in Fig. 3D. In order to decouple the rotational input $R 1$ from $R 2$, the flexures shown in Fig. 3E may also be attached. Similar flexures could be attached ontop of the system to decouple $R 2$ from $R 1$. The resulting 2 DOF flexure-based transmission mechanism is shown in Fig. 3F. 


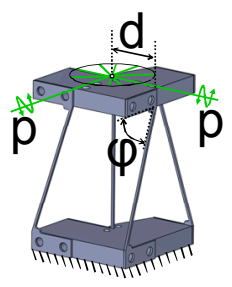

(A)

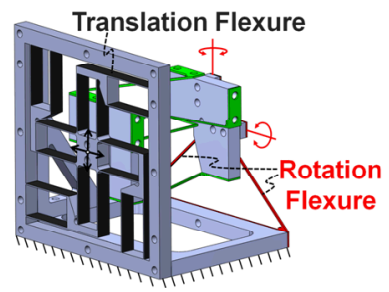

(D)

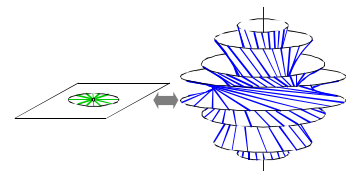

(B)

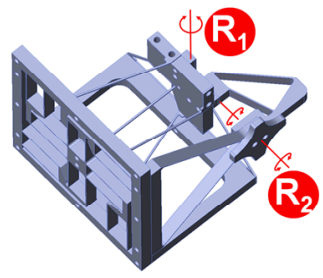

(E)

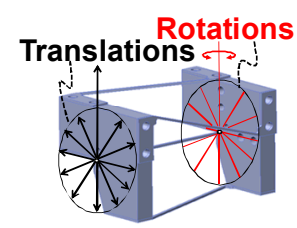

(C)

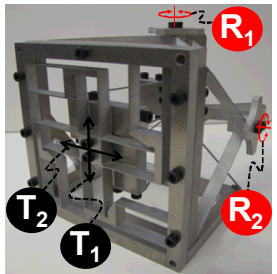

(F)

Figure 3: Synthesis of a multi-input decoupled flexure-based transmission mechanism

\section{Conclusion}

We have provided a synopsis of a method wherein designers may use geometric shapes to synthesize flexure-based transmission mechanisms that possess multiple inputs that link to decoupled outputs of various types with designer-specified transmission ratios. A flexure-based mechanism with two decoupled rotational inputs that link to two translational outputs was fabricated and tested. The predicted transmission ratio of $515 \mu \mathrm{m}$ per degree of rotation was within $8.6 \%$ error of the ratio measured using the device. This work was performed under the auspices of the U.S. Department of Energy by Lawrence Livermore National Laboratory under Contract DE-AC52-07NA27344.

\section{References:}

[1] J. Hopkins, M. Culpepper. Synthesis of Multi-degree of Freedom, Parallel Flexure System Concepts via Freedom and Constraint Topology (FACT) - Part I: Principles, Precision Engineering, 34 (2010): pp. 259-270.

[2] J. Hopkins, M. Culpepper. A Screw Theory Basis for Quantitative and Graphical Design Tools that Define Layout of Actuators to Minimize Parasitic Errors in Parallel Flexure Systems, Precision Engineering, 34 (2010): pp. 767-776. 